\title{
Características dendrométricas de eucalipto submetido a desbaste
}

\author{
Dendrometric characteristics of eucalyptus subjected to thinning
}

\author{
Leonidas Soares Murta Júnior ${ }^{1}$ (D), Renato Vinícius Oliveira Castro ${ }^{2}$ (ㄹ, Mauro Eloi Nappo $^{3}$ (i), \\ Gilciano Saraiva Nogueira ${ }^{4}$ (1), Carlos Alberto Araújo Júnior ${ }^{5}$ (1), Ricardo de Oliveira Gaspar ${ }^{3}$ (D), \\ Lidiomar Soares da Costa $^{6}$ (1) , Erica Karolina Barros de Oliveira ${ }^{3}$ (1) \\ ${ }^{1}$ Instituto Estadual de Florestas - IEF, Teófilo Otoni, MG, Brasil \\ ${ }^{2}$ Universidade Federal de São João del-Rei - UFS,, Sete Lagoas, MG, Brasil \\ ${ }^{3}$ Universidade de Brasília - UnB, Brasília, DF, Brasil \\ ${ }^{4}$ Universidade Federal dos Vales do Jequitinhonha e Mucuri - UFVJM, Diamantina, MG, Brasil \\ ${ }^{5}$ Universidade Federal de Minas Gerais - UFMG, Montes Claros, MG, Brasil \\ 6Universidade Federal de Uberlândia - UFU, Monte Carmelo, MG, Brasil
}

\begin{abstract}
Como citar: Murta Júnior, L. S., Castro, R. V. O., Nappo, M. E., Nogueira, G. S., Araújo Júnior, C. A., Gaspar, R. O., Costa, L. S., \& Oliveira, E. K. B. (2020). Características dendrométricas de eucalipto submetido a desbaste. Scientia Forestalis, 48(127), e2948. https://doi.org/10.18671/scifor.v48n127.22
\end{abstract}

\begin{abstract}
Resumo
O desbaste é uma técnica que visa a produção de madeira de alta qualidade através da obtenção de árvores de grande porte em um menor tempo, alterando as características dendrométricas das árvores remanescentes. Assim, objetivou-se apresentar as mudanças nas características dendrométricas, além de ajustar e selecionar modelos hipsométricos para um povoamento de eucalipto submetido a diferentes intensidades de desbaste sistemático. Os tratamentos foram: T0: testemunha (sem desbaste); T25: redução de $25 \%$ no número de indivíduos; T33: redução de 33\%; e T50: redução de 50\%. O povoamento foi mensurado aos 60 meses (antes da intervenção), 67, 74, 81, 88 e 95 meses. As variáveis dendrométricas avaliadas foram diâmetro a 1,30 m de altura (DAP), altura total $(H t)$, mortalidade e área basal por hectare. Os modelos hipsométricos testados foram o de Stoffels, o Modelo de Linha Reta e o de Curtis. Aplicou-se o teste de identidade de modelos para verificar a possibilidade de uma única equação representar a relação hipsométrica para os tratamentos dentro da mesma idade. O desbaste influenciou o crescimento em DAP e área basal por hectare, porém houve pouca influência no crescimento em altura total. A mortalidade foi inferior a 3,3\% para todos os tratamentos, exceto para o T50 que foi de 7\%. O modelo de Curtis resultou nas melhores estimativas de altura total. O desbaste proporcionou alterações nos incrementos diamétricos dos tratamentos resultando na impossibilidade de utilizar uma única equação hipsométrica para os diferentes tratamentos em cada idade.
\end{abstract}

Palavas-chave: Modelo hipsométrico; Teste de identidade de modelo; Espaçamento.

\begin{abstract}
Thinning is a silvicultural technique that aims to produce high quality wood by obtaining large trees in a shorter time, changing the dendrometric characteristics of the remaining trees. Thus, the objective was to present the changes in the dendrometric characteristics, besides adjusting and selecting hypsometric models for a eucalyptus stand subjected to different intensities of systematic thinning. The treatments were: T0: control (without thinning); T25: reduction of $25 \%$ in the number of individuals; T33: reduction of 33\%; and T50: 50\% reduction. The stand was measured at 60 months (before intervention), 67, 74, 81, 88 and 95 months. The dendrometric variables evaluated were diameter at breast height (DBH), total height $(\mathrm{Ht})$, mortality and basal area per hectare. The hypsometric models tested were Stoffels, Linear and Curtis Models. The model identity test was applied to verify the possibility of a single equation representing the
\end{abstract}

Fonte de financiamento: $O$ presente trabalho foi realizado com apoio da Coordenação de Aperfeiçoamento de Pessoal de Nível Superior - Brasil (CAPES) -

Código de Financiamento: 001.

Conflito de interesse: Nada a declarar.

Autor correspondente: murtaengflo@gmail.com

Recebido: 8 Julho 2018.

Aceito: 22 Outubro 2019

Editor: Paulo Henrique Müller Silva.

(c) (1) Este é um artigo publicado em acesso aberto (Open Access) sob a licença Creative Commons Attribution, que permite uso, distribuição e

c) reprodução em qualquer meio, sem restrições desde que o trabalho original seja corretamente citado. 
hypsometric relation for the treatments within the same age. Thinning influenced growth in DBH and basal area per hectare, but there was little influence on total height growth. Mortality was lower than 3.3\% for all treatments, except for T50 which was 7\%. The Curtis model resulted in the best total height estimates. Thinning provided changes in the diametric increments of the treatments resulting in the impossibility of using a single hypsometric equation for the different treatments at each age.

Keywords: Hypsometric model; Model identity test; Spacing.

\section{INTRODUÇÃO}

O comportamento de uma floresta plantada, no que se refere aos tipos diferenciados de manejo, pode proporcionar alterações na qualidade da madeira, influenciando no produto final desejado e, consequentemente, no seu valor comercial. Nesse sentido, a busca por material de qualidade superior que atenda às exigências do mercado madeireiro vem crescendo a cada ano. Tal comportamento é refletido nos diferentes segmentos florestais a partir da silvicultura e do melhoramento genético, finalizando no setor tecnológico de base florestal (Gonçalves et al., 2010).

Em plantios comerciais, diversas práticas silviculturais devem ser executadas quando o objetivo é a produção de madeira sólida de qualidade (David et al., 2017). Dentre tais atividades, o desbaste constitui-se como uma das mais importantes (Gonçalves et al., 2010). Sua aplicação pode resultar na produção de árvores de grande porte, se houver gestão dos fatores de crescimento, como água, luz e nutrientes para as árvores, com a garantia de aumento na qualidade da madeira, o que possivelmente irá agregar valor a esta (Dias et al., 2005).

Um dos aspectos que devem ser considerados quando da aplicação de desbastes é a sua intensidade, a qual afeta as características dendrométricas dos povoamentos florestais (Araújo et al., 2007) principalmente em termos do incremento diamétrico. Menores índices de densidade de indivíduos estimula a formação de fustes maiores em diâmetro e de maior valor agregado, por atenderem a usos mais nobres. Por outro lado, em povoamentos mais densos, o espaço para o crescimento radial das árvores é reduzido, tornando-as mais finas e pouco valorizadas no mercado (David et al., 2017).

Desde a implantação das primeiras florestas plantadas com o gênero Eucalyptus em território brasileiro, têm-se avaliado o comportamento das variáveis dendrométricas diante da aplicação de diferentes tratamentos silviculturais e suas implicações na produção florestal. Isso inclui os trabalhos sobre a adoção de diferentes espécies e a relação entre características dendrométricas em diferentes espaçamentos de plantio (Vidaurre et al., 2015; David et al., 2017), a influência da intensidade de desbaste nas características dendrométricas e tecnológicas (Trevisan et al., 2007), o efeito do desbaste e da fertilização na porcentagem de casca e conicidade de toras (Lima \& Garcia, 2011) e o comportamento das características dendrométricas, químicas e densidade da madeira (Hsing et al., 2016).

Estes estudos descreveram algumas tendências do crescimento da floresta em função da intensidade de desbaste e espaçamento adotado, podendo-se destacar o incremento em diâmetro, altura e volume, determinados pelo número de árvores por hectare. Tal conhecimento a cerca do comportamento de variáveis dendrométricas de espécies utilizadas em plantios comerciais em diferentes regiões proporcionam elementos técnicos que contribuem de maneira efetiva para o entendimento do processo de crescimento e produção (Hoffmann et al., 2011; Campos \& Leite, 2017; David et al., 2017), que será guia de tomada de decisão para o manejador florestal visando otimizar a produção em determinadas condições locais para as finalidades desejadas. Desta forma, a construção de base de dados experimentais consolidam conhecimentos já apontados e possibilitam sempre novas discussões e interpretações que retroalimentam o processo científico tecnológico, em especial para o manejo florestal.

Assim, diante do exposto, o presente estudo teve como objetivo apresentar as mudanças nas características dendrométricas, além de ajustar e selecionar modelos hipsométricos para um povoamento de eucalipto submetido a diferentes intensidades de desbaste. 


\section{MATERIAL E MÉTODOS}

O estudo foi conduzido na Fazenda Água Limpa (FAL), pertencente à Universidade de Brasília (UnB), Distrito Federal. A FAL está localizada entre as coordenadas geográficas $15^{\circ} 56^{\prime}-15^{\circ} 59^{\prime} \mathrm{S}$ e $47^{\circ} 55^{\prime}-47^{\circ} 56^{\prime} \mathrm{W}$, e encontra-se a uma altitude média de $1.100 \mathrm{~m}$. O clima da região, segundo Köppen, é do tipo Aw (Alvares et al., 2013), sendo caracterizado por duas estações bem definidas, uma quente e chuvosa que ocorre no período de outubro a abril, e outra fria e seca, que acontece de maio a setembro. A temperatura anual varia de $12,0^{\circ} \mathrm{C}$ a $28,5^{\circ} \mathrm{C}$ com precipitação média anual de $1.600 \mathrm{~mm}$ (Nimer, 1989). O solo predominante na região é o Latossolo Vermelho Amarelo, distrófico, com alto teor de alumínio e baixos teores de cálcio e magnésio (Haridasan, 1990).

Os dados foram coletados em um povoamento florestal com aproximadamente 2,10 hectares. O material genético é composto por híbrido clonal de Eucalyptus urophylla $\mathrm{x}$ Eucalyptus grandis implantado com área vital de 6,0 m² (3,0 m x 2,0 m) por planta.

Aos 60 meses foi realizado o desbaste sistemático de árvores dentro das linhas. Para isso, a área de estudo foi dividida em quatro partes de mesmo tamanho e os tratamentos aplicados corresponderam a diferentes porcentagens de redução no número de indivíduos, sendo: T0: testemunha (não houve desbaste); T25: redução de $25 \%$ no número de indivíduos; T33: redução de 33\% no número de indivíduos; e T50: redução de 50\% no número de indivíduos.

A primeira coleta de dados ocorreu aos 60 meses (antes da intervenção) e as demais aos $67,74,81,88$ e 95 meses. As variáveis coletadas foram: o diâmetro a 1,30 m de altura (DAP) de todos os indivíduos e altura total $(H t)$ de 50 árvores para cada tratamento, selecionadas aleatoriamente. As alturas foram mensuradas apenas a partir dos 67 meses, sendo que as mesmas árvores foram remedidas em cada idade. O DAP foi mensurado com suta e $\mathrm{Ht}$ com auxílio de clinômetro digital.

A dinâmica do povoamento no período estudado foi calculada com base nas variações obtidas no crescimento em $D A P$ e a $H t$, na mortalidade dos indivíduos e na área basal. As variações foram calculadas por tratamento, em escala absoluta e percentual.

Para estimar a altura total das árvores que não tiveram esta variável mensurada, foram ajustados modelos hipsométricos por estrato (idade e tratamento), totalizando 20 ajustes por modelo. Os modelos avaliados foram selecionados com base na literatura florestal pertinente (Stoffels \& Soest, 1953; Curtis, 1967; Soares et al., 2004, 2011; Campos \& Leite, 2017) e estão apresentados na Tabela 1. Embora a relação matemática entre o diâmetro e altura total das árvores não seja linear, o Modelo de linha reta também foi testado conforme trabalhos de Soares et al. (2004), Donadoni et al. (2010) e Azevedo et al. (2011).

Tabela 1. Modelos avaliados para estimar a altura das árvores por idade e tratamento

\begin{tabular}{cccc}
$\mathbf{N}^{\circ}$ & Forma de ajuste & Autor & \\
\hline 1 & $L n H t$ & $=\beta_{0}+\beta_{1} L n D A P+\varepsilon$ & Stoffels
\end{tabular}

em que: $L n$ é o logaritmo neperiano; $H t$ é a altura total $(\mathrm{m}) ; \beta_{0}$ e $\beta_{I}$ são parâmetros dos modelos; DAP é o diâmetro a $1,3 \mathrm{~m}$ de altura (cm); e $\varepsilon$ é o erro aleatório.

Das 50 observações em cada estrato, $60 \%$ foram destinados para o ajuste dos modelos e $40 \%$ para a fase de validação das equações. Para garantir maior amplitude aos dados de ajuste, as árvores foram distribuídas em cinco classes de altura, e em cada classe selecionou-se aleatoriamente seis observações. Nas situações em que a classe apresentou seis ou menos árvores, todas elas foram direcionadas para o grupo de ajuste. 
Para verificar a qualidade dos ajustes, bem como as estimativas de $H t$ obtidas com cada equação, avaliou-se os valores do coeficiente de determinação $\left(R^{2}\right)$, erro-padrão residual $\left(S_{x y}\right)$, coeficiente de correlação de Pearson $(r)$, raiz quadrada do erro médio (\%), Bias absoluto e percentual, média das diferenças absolutas e análise gráfica de resíduos (dispersão, gráfico de 45 graus e histograma dos resíduos percentuais) cujas relações funcionais estão descritas em Ozçelik et al. (2010), Binoti et al. (2015) e Campos \& Leite (2017).

As equações que permitiram obter as melhores estimativas aos 67 meses foram utilizadas, em seus respectivos tratamentos, para estimar a altura total das árvores aos 60 meses.

Após selecionar o melhor modelo e ajustar as equações hipsométricas para cada tratamento por idade, aplicou-se o teste de identidade de modelos (Graybill, 1976), utilizando variáveis dummy (Regazzi, 2003) com o objetivo de verificar a possibilidade de uma única equação representar a relação hipsométrica para os tratamentos dentro da mesma idade. Este teste compara a soma de quadrado dos resíduos em cada tratamento (modelo completo) e a soma de quadrado da diferença para o modelo ajustado com todos os tratamentos (modelo reduzido). A hipótese de nulidade testada $\left(\mathrm{H}_{0}\right)$, foi a de que os parâmetros eram iguais, ou seja, que o vetor dos parâmetros do modelo completo fosse igual ao do modelo reduzido. A rejeição dessa hipótese implica na impossibilidade do uso de uma única equação para estimar a altura total das árvores entre os tratamentos testados.

Todas as combinações entre os quatro tratamentos foram testadas e a verificação da hipótese $\mathrm{H}_{0}$ foi realizada através do teste $\mathrm{F}$ da análise de variância para a redução, considerando o nível de significância de 5\% (Neter et al., 1996; Araújo et al., 2012).

\section{RESULTADOS E DISCUSSÃO}

A descrição completa das variáveis diâmetro $(D A P)$, altura total $(H t)$, diâmetro médio $(q)$ e área basal por hectare $(A B)$, para cada tratamento e em cada idade, além do número de indivíduos colhidos na implantação do desbaste sistemático, são apresentados na Tabela 2.

Aos 60 meses, nota-se que a divisão do povoamento resultou em quatro tratamentos homogêneos, sendo que os valores médios das variáveis dendrométricas foram similares. A exceção foi o T33, que apresentou os menores valores para as variáveis analisadas. Nos demais tratamentos as médias de DAP ficaram em torno de $15,5 \mathrm{~cm}$, as de $H t$ em cerca de $21,8 \mathrm{~m}$, o diâmetro médio em 15,6 cm e as áreas basais foram superiores a $20 \mathrm{~m}^{2} \cdot \mathrm{ha}^{-1}$.

A aplicação do desbaste resultou na colheita de 117, 185 e 262 árvores para os tratamentos T25, T33 e T50, respectivamente. Embora buscou-se implantar exatamente os percentuais de redução no número de árvores nos tratamentos, as intensidades de desbaste reais foram de 20,5\% para o T25, 33,2\% para o T33 e 43,9\% para o T50. O impacto da implantação dos tratamentos foi discreto nos valores das variáveis $D A P, H t$ e $q$, os quais praticamente não alteraram seus valores. Já para a área basal, a remoção das árvores resultou numa redução de 20,1\% no T25; 33,4\% no $T 33$ e 42,5\% para o tratamento $T 50$.

Aos 95 meses percebe-se que as árvores atingiram, em termos médios, diâmetros superiores aos 19,0 cm nos tratamentos $T 25$ e T50, assim como para o diâmetro médio $(q)$. Já para a altura total, as maiores médias foram constatadas nos tratamentos T0 e T25, respectivamente.

As mudanças no povoamento em termos de dinâmica de crescimento diamétrico, crescimento em altura total, mortalidade e área basal ocorridas a partir da realização do desbaste são apresentadas na Tabela 3. 
Tabela 2. Descrição dos dados do povoamento de eucalipto submetido a diferentes intensidades de desbaste, localizado em Brasília-DF

\begin{tabular}{|c|c|c|c|c|c|c|c|c|c|c|c|c|c|c|c|c|}
\hline \multirow{2}{*}{ Variáveis } & \multicolumn{4}{|c|}{60 meses } & \multicolumn{4}{|c|}{60 meses ** } & \multicolumn{4}{|c|}{67 meses } & \multicolumn{4}{|c|}{74 meses } \\
\hline & то & T25 & T33 & T50 & то & T25 & T33 & T50 & то & T25 & T33 & T50 & то & T25 & T33 & T50 \\
\hline DAP mín (cm) & 5,60 & 5,90 & 4,00 & 6,40 & 5,60 & 5,90 & 4,00 & 6,40 & 5,60 & 7,00 & 4,80 & 6,70 & 8,50 & 6,60 & 5,60 & 6,90 \\
\hline DAP méd $(\mathrm{cm})$ & 15,59 & 15,59 & 14,78 & 15,43 & 15,59 & 15,63 & 14,74 & 15,60 & 16,80 & 16,92 & 16,22 & 17,15 & 17,38 & 17,58 & 16,88 & 17,93 \\
\hline DAP máx $(\mathrm{cm})$ & 22,50 & 21,00 & 24,60 & 22,60 & 22,50 & 21,00 & 24,60 & 22,60 & 24,30 & 22,50 & 25,30 & 24,80 & 24,80 & 23,50 & 28,20 & 25,60 \\
\hline Ht mín $(m)^{*}$ & 10,13 & 5,87 & 4,88 & 13,08 & 10,13 & 5,87 & 4,88 & 13,08 & 10,13 & 8,19 & 6,72 & 13,62 & 16,48 & 5,94 & 10,96 & 13,57 \\
\hline$H t$ méd $(m)^{*}$ & 22,05 & 21,68 & 19,43 & 21,84 & 22,05 & 21,72 & 19,40 & 21,91 & 22,77 & 23,11 & 20,41 & 22,68 & 25,20 & 24,17 & 21,72 & 23,71 \\
\hline$H t$ máx $(\mathrm{m})^{*}$ & 25,43 & 26,92 & 24,29 & 24,78 & 25,43 & 26,92 & 24,29 & 24,78 & 26,60 & 28,01 & 25,60 & 25,40 & 28,75 & 30,20 & 26,10 & 26,60 \\
\hline$q(\mathrm{~cm})$ & 15,74 & 15,74 & 15,03 & 15,62 & 15,74 & 15,78 & 15,00 & 15,81 & 16,98 & 17,08 & 16,47 & 17,38 & 17,54 & 17,74 & 17,14 & 18,16 \\
\hline$A B\left(\mathrm{~m}^{2} . \mathrm{ha}^{-1}\right)$ & 20,06 & 21,17 & 18,75 & 21,13 & 20,06 & 16,91 & 12,49 & 12,16 & 22,98 & 19,50 & 14,89 & 13,88 & 24,22 & 21,01 & 16,05 & 14,99 \\
\hline $\mathbf{N}^{\circ}$ de indivíduos desbastados & 0 & 117 & 185 & 262 & - & - & - & - & - & - & - & - & & - & - & \\
\hline \multirow{2}{*}{ Variáveis } & \multicolumn{4}{|c|}{81 meses } & \multicolumn{4}{|c|}{88 meses } & \multicolumn{4}{|c|}{95 meses } & & & & \\
\hline & Tо & T25 & T33 & T50 & то & T25 & T33 & T50 & то & T25 & T33 & T50 & & & & \\
\hline DAP mín (cm) & 9,00 & 8,10 & 6,00 & 6,90 & 9,60 & 8,40 & 6,40 & 6,90 & 9,70 & 7,80 & 6,70 & 6,90 & & & & \\
\hline$D A P$ méd $(\mathrm{cm})$ & 17,88 & 18,26 & 17,58 & 18,66 & 18,38 & 18,92 & 18,24 & 19,35 & 18,47 & 19,04 & 18,42 & 19,55 & & & & \\
\hline DAP máx $(\mathrm{cm})$ & 25,80 & 25,00 & 30,10 & 27,00 & 27,00 & 26,30 & 31,60 & 28,50 & 27,50 & 26,50 & 32,40 & 29,60 & & & & \\
\hline$H t$ mín $(m)^{*}$ & 17,63 & 11,36 & 11,50 & 13,34 & 18,81 & 13,56 & 11,40 & 12,58 & 18,69 & 11,82 & 11,37 & 12,47 & & & & \\
\hline$H t$ méd $(m)^{*}$ & 25,76 & 25,32 & 22,16 & 24,06 & 26,22 & 25,80 & 22,73 & 24,70 & 26,69 & 26,17 & 23,20 & 25,02 & & & & \\
\hline$H t$ máx $(m)^{*}$ & 29,25 & 30,45 & 26,20 & 27,06 & 29,70 & 30,12 & 26,97 & 28,18 & 30,66 & 30,90 & 28,10 & 28,77 & & & & \\
\hline$q(\mathrm{~cm})$ & 18,07 & 18,45 & 17,87 & 18,92 & 18,57 & 19,12 & 18,54 & 19,61 & 18,66 & 19,25 & 18,72 & 19,82 & & & & \\
\hline$A B\left(\mathrm{~m}^{2} . \mathrm{ha}^{-1}\right)$ & 25,69 & 22,61 & 17,43 & 16,27 & 26,99 & 24,22 & 18,77 & 17,42 & 27,23 & 24,55 & 19,09 & 17,74 & & & & \\
\hline
\end{tabular}

em que: $D A P$ é o diâmetro à 1,30 m de altura $(\mathrm{cm})$; mín é mínimo; méd é médio; máx é máximo; $H t$ é a altura total $(\mathrm{m})$; q é o diâmetro médio $(\mathrm{cm})$; e $A B$ é a área basal $\left(\mathrm{m}^{2} \cdot \mathrm{ha}^{-1}\right)$. * dados estimados através de modelos hipsométricos; ** Após a realização do desbaste.

Os tratamentos T33 e T50, que receberam as maiores intensidades de desbaste, proporcionaram os maiores crescimentos em diâmetro e área basal. As taxas de incremento para essas variáveis foram maiores no primeiro intervalo de medição (60 aos 67 meses), em que seus valores foram superiores a $7 \%$ para DAP e $14 \%$ para área basal por hectare. No último intervalo de medição (88 a 95 meses), o crescimento diamétrico e em área basal nestes tratamentos foram o dobro do observado no tratamento testemunha.

Já para a altura total percebe-se pouca influência do efeito do desbaste no seu ritmo de crescimento. Nota-se uma pequena superioridade nas médias dos crescimentos no período de 67 a 74 meses em relação aos demais, onde a testemunha apresentou um incremento superior a 10\%. Em cada período, tratamentos distintos apresentaram maiores médias de crescimento, não sendo possível indicar qual proporcionou maior influência no crescimento em altura total das árvores.

A mortalidade percentual na maioria dos períodos foi inferior a $1 \%$ para todos os tratamentos. Apenas no período de 60 a 67 meses notou-se um número mais elevado de indivíduos mortos, sobretudo para o tratamento $T 50$, em que o valor chegou a 5,54\%.

Após 35 meses da implantação do desbaste sistemático, o tratamento que resultou no maior crescimento em $D A P$ e em área basal foi o T33, proporcionando um incremento diamétrico maior que $25 \%$ contra aproximadamente $17 \%$ do tratamento testemunha. Em termos de área basal, esse incremento médio foi superior a 50\% contra 35\% do tratamento sem desbaste. Para a altura total, os tratamentos apresentaram crescimentos médios superiores à $20 \%$, exceto o T50 em que observou-se um crescimento inferior, próximo aos 
13\%. Considerando a mortalidade, os tratamentos que apresentaram maiores taxas foram T50, seguido de TO, T25 e T33, com valores iguais a 7,1\%; 3,3\%; 2,4\% e 1,9\%, respectivamente (Tabela 4).

Tabela 3. Mudanças ocorridas por período no povoamento de eucalipto submetido a diferentes intensidades de desbaste, localizado em Brasília-DF

\begin{tabular}{|c|c|c|c|c|c|c|c|c|c|c|c|c|}
\hline \multirow{2}{*}{ Variáveis } & \multicolumn{4}{|c|}{$60 * *$ a 67 meses } & \multicolumn{4}{|c|}{67 a 74 meses } & \multicolumn{4}{|c|}{74 a 81 meses } \\
\hline & TO & $T 25$ & T33 & $T 50$ & TO & $T 25$ & T33 & T50 & TO & $T 25$ & T33 & T50 \\
\hline$\triangle D A P(\mathrm{~cm})$ & 1,16 & 1,24 & 1,44 & 1,39 & 0,49 & 0,65 & 0,65 & 0,71 & 0,50 & 0,65 & 0,70 & 0,73 \\
\hline$\triangle D A P(\%)$ & 7,36 & 7,97 & 10,19 & 8,83 & 2,88 & 3,85 & 3,99 & 4,16 & 2,82 & 3,60 & 4,18 & 4,04 \\
\hline$\Delta H t(\mathrm{~m})^{*}$ & 0,68 & 1,33 & 0,97 & 0,68 & 2,35 & 1,05 & 1,31 & 0,98 & 0,56 & 1,09 & 0,44 & 0,35 \\
\hline$\Delta H t(\%)^{*}$ & 3,11 & 6,31 & 5,38 & 3,13 & 10,32 & 4,30 & 6,82 & 4,31 & 2,24 & 4,97 & 2,03 & 1,46 \\
\hline Mortalidade ( ${ }^{\circ}$ de indivíduos) & 8 & 7 & 4 & 18 & 7 & 1 & 2 & 3 & 0 & 2 & 0 & 0 \\
\hline Mortalidade (\%) & 1,48 & 1,54 & 1,08 & 5,54 & 1,31 & 0,22 & 0,54 & 0,98 & 0,00 & 0,45 & 0,00 & 0,00 \\
\hline$\Delta A B\left(\mathrm{~m}^{2} . \mathrm{ha}^{-1}\right)$ & 2,92 & 2,59 & 2,40 & 1,72 & 1,24 & 1,50 & 1,16 & 1,12 & 1,47 & 1,60 & 1,38 & 1,28 \\
\hline$\triangle A B(\%)$ & 14,56 & 15,34 & 19,20 & 14,11 & 5,40 & 7,71 & 7,79 & 8,05 & 6,07 & 7,62 & 8,62 & 8,54 \\
\hline \multirow{2}{*}{ Variáveis } & \multicolumn{4}{|c|}{81 a 88 meses } & \multicolumn{4}{|c|}{88 a 95 meses } & & & & \\
\hline & TO & $T 25$ & $T 33$ & $T 50$ & TO & $T 25$ & T33 & T50 & & & & \\
\hline$\triangle D A P(\mathrm{~cm})$ & 0,47 & 0,63 & 0,66 & 0,66 & 0,08 & 0,12 & 0,16 & 0,19 & & & & \\
\hline$\triangle D A P(\%)$ & 2,59 & 3,41 & 3,79 & 3,57 & 0,45 & 0,63 & 0,91 & 0,98 & & & & \\
\hline$\Delta H t(\mathrm{~m})^{\star}$ & 0,44 & 0,45 & 0,57 & 0,63 & 0,46 & 0,37 & 0,47 & 0,31 & & & & \\
\hline$\Delta H t(\%)^{*}$ & 1,74 & 2,01 & 2,50 & 2,58 & 1,73 & 1,35 & 1,99 & 1,24 & & & & \\
\hline Mortalidade ( $\mathrm{n}^{\circ}$ de indivíduos) & 3 & 1 & 0 & 1 & 0 & 0 & 1 & 1 & & & & \\
\hline Mortalidade (\%) & 0,57 & 0,23 & 0,00 & 0,33 & 0,00 & 0,00 & 0,27 & 0,33 & & & & \\
\hline$\triangle A B\left(\mathrm{~m}^{2} . \mathrm{ha}^{-1}\right)$ & 1,30 & 1,62 & 1,34 & 1,15 & 0,25 & 0,32 & 0,32 & 0,32 & & & & \\
\hline$\triangle A B(\%)$ & 5,04 & 7,14 & 7,70 & 7,07 & 0,91 & 1,34 & 1,70 & 1,84 & & & & \\
\hline
\end{tabular}

em que: $\triangle D A P$ é a média do crescimento em $D A P(\mathrm{~cm}) ; \Delta H t$ é a média do crescimento em altura total $(\mathrm{m}) ; \triangle A B$ é o crescimento em área basal $\left(\mathrm{m}^{2} . \mathrm{ha}^{-1}\right)$. * dados estimados através de modelos hipsométricos; ** Após a realização do desbaste.

Tabela 4. Mudanças ocorridas no povoamento de eucalipto após 35 meses da implantação dos desbastes, em Brasília-DF

\begin{tabular}{|c|c|c|c|c|}
\hline \multirow{2}{*}{ Variáveis } & \multicolumn{4}{|c|}{60 ** a 95 meses } \\
\hline & TO & $T 25$ & T33 & T50 \\
\hline$\triangle D A P(\mathrm{~cm})$ & 2,72 & 3,31 & 3,63 & 3,71 \\
\hline$\triangle D A P(\%)$ & 17,15 & 20,94 & 25,35 & 23,54 \\
\hline$\Delta H t(\mathrm{~m})^{\star}$ & 4,51 & 4,30 & 3,75 & 2,96 \\
\hline$\Delta H t(\%)^{*}$ & 20,33 & 20,22 & 20,18 & 13,37 \\
\hline Mortalidade ( $\mathrm{n}^{\circ}$ de indivíduos) & 18 & 11 & 7 & 23 \\
\hline Mortalidade (\%) & 3,33 & 2,42 & 1,89 & 7,08 \\
\hline$\triangle A B\left(\mathrm{~m}^{2} . \mathrm{ha}^{-1}\right)$ & 7,17 & 7,64 & 6,60 & 5,58 \\
\hline$\triangle A B(\%)$ & 35,76 & 45,17 & 52,86 & 45,91 \\
\hline
\end{tabular}

em que: $\triangle D A P$ é a média do crescimento em $D A P(\mathrm{~cm}) ; \Delta H t$ é a média do crescimento em altura total $(\mathrm{m}) ; \triangle A B$ é o crescimento em área basal $\left(\mathrm{m}^{2} \cdot \mathrm{ha}^{-1}\right)$. * dados estimados através de modelos hipsométricos; ${ }^{* *}$ Após a realização do desbaste. 
Os parâmetros e as estatísticas de qualidade das estimativas obtidas nas fases de ajuste e validação provenientes dos três modelos hipsométricos ajustados por idade e tratamento são apresentados na Tabela 5. Todos os parâmetros obtidos no ajuste dos modelos foram significativos pelo Teste t à $95 \%$ de probabilidade.

Tabela 5. Parâmetros das equações hipsométricas ajustadas e suas respectivas estatísticas de qualidade obtidas nas fases de ajuste e validação em povoamento de eucalipto submetido a desbaste, localizado em Brasília-DF

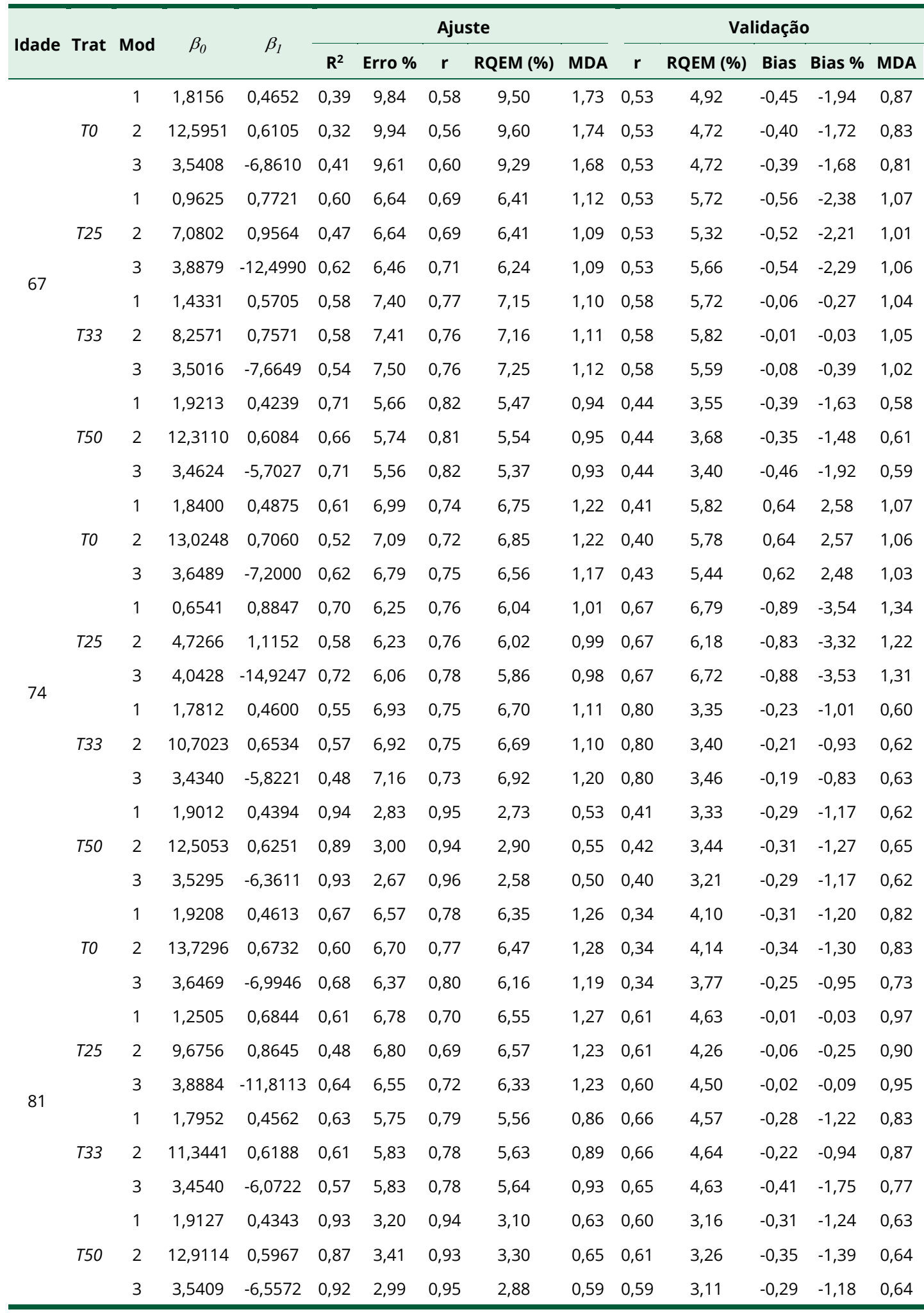


Tabela 5. Continuação..

\begin{tabular}{|c|c|c|c|c|c|c|c|c|c|c|c|c|c|c|}
\hline \multirow{2}{*}{ Idade } & \multirow{2}{*}{ Trat } & \multirow{2}{*}{ Mod } & \multirow{2}{*}{$\beta_{0}$} & \multirow{2}{*}{$\beta_{1}$} & \multicolumn{5}{|c|}{ Ajuste } & \multicolumn{5}{|c|}{ Validação } \\
\hline & & & & & $\mathbf{R}^{2}$ & Erro \% & $r$ & RQEM (\%) & MDA & $r$ & RQEM (\%) & Bias & Bias \% & MDA \\
\hline \multirow{11}{*}{88} & & 1 & 1,9576 & 0,4504 & 0,74 & 5,38 & 0,84 & 5,20 & 1,02 & 0,43 & 4,74 & $-0,59$ & $-2,20$ & 0,94 \\
\hline & TO & 2 & 13,7053 & 0,6817 & 0,70 & 5,43 & 0,84 & 5,24 & 1,04 & 0,42 & 4,90 & $-0,61$ & $-2,25$ & 0,98 \\
\hline & & 3 & 3,6428 & $-6,7981$ & 0,72 & 5,40 & 0,84 & 5,21 & 1,00 & 0,44 & 4,28 & $-0,54$ & $-2,02$ & 0,82 \\
\hline & & 1 & 1,6263 & 0,5543 & 0,59 & 6,38 & 0,70 & 6,17 & 1,25 & 0,60 & 3,99 & $-0,34$ & $-1,28$ & 0,78 \\
\hline & $T 25$ & 2 & 12,6693 & 0,6996 & 0,47 & 6,45 & 0,68 & 6,23 & 1,22 & 0,60 & 3,88 & $-0,38$ & $-1,42$ & 0,74 \\
\hline & & 3 & 3,7794 & $-9,8446$ & 0,62 & 6,17 & 0,72 & 5,96 & 1,20 & 0,60 & 3,81 & $-0,32$ & $-1,21$ & 0,73 \\
\hline & & 1 & 1,7999 & 0,4573 & 0,68 & 5,64 & 0,79 & 5,45 & 0,95 & 0,63 & 4,87 & $-0,42$ & $-1,74$ & 0,88 \\
\hline & T33 & 2 & 12,3197 & 0,5737 & 0,61 & 5,80 & 0,78 & 5,61 & 0,97 & 0,63 & 4,93 & $-0,42$ & $-1,74$ & 0,90 \\
\hline & & 3 & 3,5133 & $-6,9089$ & 0,66 & 5,47 & 0,81 & 5,28 & 0,88 & 0,64 & 4,85 & $-0,44$ & $-1,86$ & 0,88 \\
\hline & & 1 & 1,8480 & 0,4603 & 0,90 & 3,88 & 0,92 & 3,75 & 0,70 & 0,58 & 2,75 & $-0,12$ & $-0,45$ & 0,55 \\
\hline & $T 50$ & 2 & 12,8651 & 0,6135 & 0,82 & 4,10 & 0,91 & 3,96 & 0,72 & 0,58 & 2,83 & $-0,15$ & $-0,59$ & 0,56 \\
\hline \multirow{13}{*}{95} & & 3 & 3,5964 & $-7,3417$ & 0,91 & 3,56 & 0,93 & 3,44 & 0,62 & 0,58 & 2,69 & $-0,13$ & $-0,52$ & 0,55 \\
\hline & & 1 & 1,8678 & 0,4868 & 0,76 & 5,46 & 0,85 & 5,28 & 1,04 & 0,43 & 4,75 & $-0,45$ & $-1,64$ & 0,94 \\
\hline & TO & 2 & 13,1045 & 0,7376 & 0,71 & 5,52 & 0,84 & 5,33 & 1,06 & 0,42 & 4,87 & $-0,46$ & $-1,67$ & 0,97 \\
\hline & & 3 & 3,6928 & $-7,4186$ & 0,73 & 5,44 & 0,85 & 5,26 & 1,03 & 0,43 & 4,28 & $-0,42$ & $-1,52$ & 0,84 \\
\hline & & 1 & 1,5036 & 0,5995 & 0,72 & 5,16 & 0,80 & 4,98 & 1,05 & 0,53 & 5,29 & $-0,43$ & $-1,59$ & 1,07 \\
\hline & $T 25$ & 2 & 11,2142 & 0,7917 & 0,62 & 5,23 & 0,79 & 5,05 & 1,04 & 0,53 & 5,09 & $-0,42$ & $-1,57$ & 1,03 \\
\hline & & 3 & 3,8315 & $-10,6234$ & 0,74 & 4,94 & 0,82 & 4,77 & 1,01 & 0,52 & 5,08 & $-0,41$ & $-1,53$ & 1,00 \\
\hline & & 1 & 1,6450 & 0,5163 & 0,72 & 5,57 & 0,83 & 5,38 & 1,02 & 0,65 & 4,68 & 0,01 & 0,05 & 0,76 \\
\hline & T33 & 2 & 10,9232 & 0,6705 & 0,67 & 5,68 & 0,82 & 5,49 & 1,06 & 0,65 & 4,79 & 0,03 & 0,13 & 0,78 \\
\hline & & 3 & 3,5716 & $-7,6414$ & 0,66 & 5,57 & 0,83 & 5,38 & 0,98 & 0,64 & 4,33 & $-0,04$ & $-0,15$ & 0,70 \\
\hline & & 1 & 1,8171 & 0,4733 & 0,93 & 3,20 & 0,94 & 3,09 & 0,56 & 0,55 & 3,69 & $-0,50$ & $-1,91$ & 0,79 \\
\hline & T50 & 2 & 12,4333 & 0,6462 & 0,88 & 3,36 & 0,94 & 3,25 & 0,58 & 0,55 & 3,85 & $-0,51$ & $-1,94$ & 0,83 \\
\hline & & 3 & 3,6135 & $-7,5238$ & 0,93 & 2,98 & 0,95 & 2,88 & 0,54 & 0,54 & 3,56 & $-0,54$ & $-2,07$ & 0,77 \\
\hline
\end{tabular}

em que Trat é tratamento; Mod é o número do modelo; $\beta_{0}$ e $\beta_{1}$ são parâmetros do modelo; $R^{2}$ é o coeficiente de determinação; Erro (\%) é o erro-padrão percentual; r é o coeficiente de correlação de Pearson; RQEM é a raiz quadrada do erro médio percentual; Bias é o viés, em m e percentual; e, MDA é a média das diferenças absolutas (m). Obs.: Todos os parâmetros dos modelos foram significativos pelo Teste tà 95\% de probabilidade.

Para selecionar as melhores equações, fez-se a análise gráfica dos resíduos percentuais, conforme sugerido por Campos \& Leite (2017). Os gráficos mostraram que os modelos proporcionaram resíduos não tendenciosos, homocedásticos, valores estimados e observados tendendo a uma reta 1:1 e histogramas de frequência tendendo a curva normal com média em zero, indicando que a pressuposição de normalidade dos erros foi atendida (Campos \& Leite, 2017).

O modelo de Curtis (3) foi superior aos demais devido à menor dispersão dos resíduos nas estimativas destinadas à validação dos modelos, confirmando a tendência de superioridade indicada nas estatísticas de qualidade. Gráficos de resíduos obtidos para este modelo estão apresentados na Figura 1.

Os resultados do teste de identidade de modelo para cada tratamento nas diferentes idades são apresentados na Tabela 6.

O teste foi não significativo para as comparações entre os tratamentos TO e T25; TO e T50 aos 67 meses e, TO e T25 aos 88 meses. Nos demais casos, o teste significativo indicou que não é possível utilizar apenas uma equação para estimar a altura total das árvores nas referidas idades. 


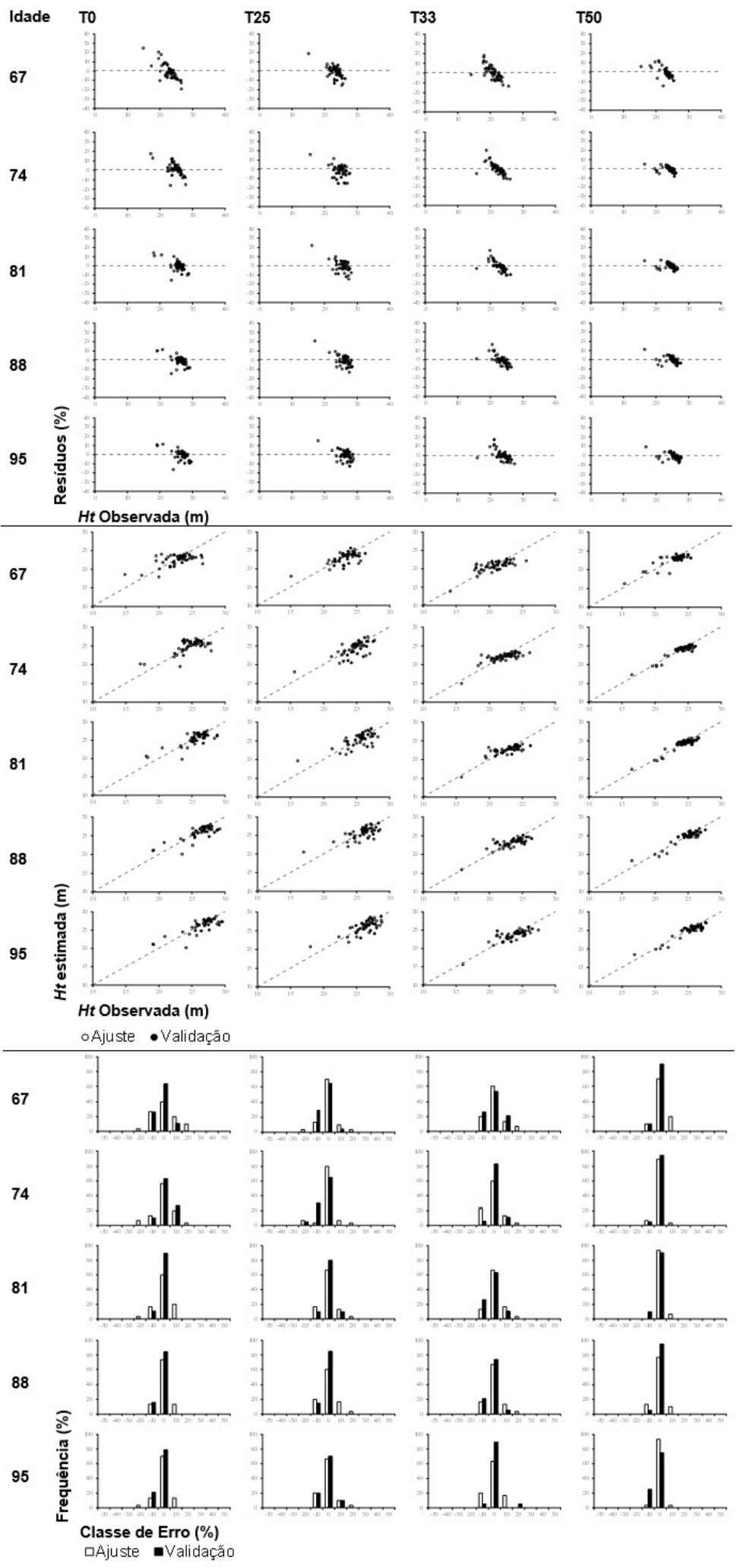

Figura 1. Análise gráfica de resíduos para o Modelo de Curtis (3) nas fases de ajuste e validação.

Os desbastes objetivam estimular o crescimento das árvores remanescentes, pois permitem a distribuição do potencial produtivo do povoamento para um número menor de indivíduos (Hawley \& Smith, 1972). Assim após a implantação desta prática silvicultural, as árvores remanescentes apresentam aumento na taxa de crescimento devido à redução de 
competição entre elas e, consequentemente, aumento na disponibilidade de luz, água e nutrientes proporcionada pelo novo espaçamento entre plantas (Leite et al., 2005; Monte et al., 2009; Hawthorne et al., 2013).

Os resultados obtidos neste estudo demonstram que as taxas de crescimento apresentaram correlação positiva com a quantidade de árvores removidas, que por consequência, reduz a competição. Os tratamentos T33 e T50, que receberam as maiores intensidades de desbaste, foram os que proporcionaram as maiores taxas de crescimento conforme Tabelas 2, 3 e 4, mostrando que quanto menor a competição restante, melhor foi a resposta obtida no crescimento. Os trabalhos de Trevisan et al. (2007), Kariuki (2008), Monte et al. (2009), Ramos et al. (2014) e Soares et al. (2017), encontraram resultados similares aos deste estudo.

Tabela 6. Resultado do teste de identidade de modelo hipsométrico para as combinações entre os diferentes tratamentos em cada idade, em Brasília-DF

\begin{tabular}{|c|c|c|c|c|}
\hline Idade & Tratamento & TO & $T 25$ & $T 33$ \\
\hline \multirow{3}{*}{67 meses } & $T 25$ & $1,21^{\mathrm{ns}}$ & & \\
\hline & T33 & $5,31 *$ & $9,56 *$ & \\
\hline & $T 50$ & $0,20 \mathrm{~ns}$ & $3,67 *$ & $8,76^{*}$ \\
\hline \multirow{3}{*}{74 meses } & $T 25$ & $6,30 *$ & & \\
\hline & T33 & $16,34^{*}$ & $12,42^{*}$ & \\
\hline & T50 & $7,76 *$ & $9,04 *$ & $6,79 *$ \\
\hline \multirow{3}{*}{81 meses } & $T 25$ & $3,03 *$ & & \\
\hline & T33 & $23,46^{*}$ & $15,50 *$ & \\
\hline & T50 & $11,33^{*}$ & $6,76^{*}$ & $7,97 *$ \\
\hline \multirow{3}{*}{88 meses } & $T 25$ & $2,40^{\mathrm{ns}}$ & & \\
\hline & T33 & $27,75^{*}$ & $14,95 *$ & \\
\hline & $T 50$ & $12,06^{*}$ & $4,02^{\star}$ & $7,68 *$ \\
\hline \multirow{3}{*}{95 meses } & $T 25$ & $3,41 *$ & & \\
\hline & T33 & $26,00 *$ & $17,10 *$ & \\
\hline & T50 & $15,33^{*}$ & $7,96 *$ & $5,57^{*}$ \\
\hline
\end{tabular}

Valor de $\mathrm{F}$ tabelado $(3 ; 56)=2,77 ;{ }^{\text {ns }}=$ não significativo ; * = significativo a 5\% de significância.

Em resposta ao desbaste, as árvores remanescentes retomam o crescimento em diâmetro (Carneiro et al., 2012; Glencross et al., 2014), o que resulta no ingresso dos indivíduos em classes de diâmetro superiores (Ramos et al., 2014). Neste estudo pode-se inferir que o diâmetro e a área basal foram as variáveis mais influenciadas pelo desbaste, sendo elas fortemente correlacionadas.

O tratamento $T 50$ resultou em maior média de $D A P$, sendo igual a 19,55 cm, e $T 33$ foi o que proporcionou maior incremento diamétrico e em área basal. Ramos et al. (2014), estudando o efeito de desbaste sobre o crescimento de $E$. grandis em Abaeté-MG, encontraram um DAP médio de 19,1 cm aos 141 meses para o tratamento de $70 \%$ de desbaste contra 16,6 cm do tratamento não desbastado. Trevisan et al. (2007) encontraram incrementos diamétrico de 38\% no tratamento com desbaste de $70 \%$ de área basal em povoamento de Eucalyptus grandis em Capivari do Sul-RS.

Glufke et al. (1997) explicam que os desbastes até certa intensidade, aumentam a área basal individual pois proporcionam maior disponibilidade de luz e nutrientes, de forma que o crescimento das árvores remanescentes compensa a retirada de árvores menores, mantendo a área basal total da floresta quase inalterada, obtendo-se ao final, árvores de maiores diâmetros. 
Para a variável altura total, quando se analisa os resultados por período (Tabela 3), notase que tratamentos distintos apresentam as maiores médias de crescimento, o que evidencia o fato do desbaste não influenciar o crescimento em altura total das árvores (Assmann, 1961; Husch et al., 2003; Sullivan et al., 2006; Monte et al., 2009).

O tratamento $T 50$ proporcionou as menores taxas de incremento em altura (13\%), já para o tratamento testemunha o crescimento foi de cerca de 20,30\%. Monte et al. (2009), obtiveram as menores alturas com o desbaste de 35\%, em comparação à testemunha um ano após o desbaste, eles concluíram que a maior altura das plantas do povoamento sem desbaste é devido à maior competição principalmente por luz, em razão do maior número de indivíduos por hectare, que estimula o crescimento em altura.

Hawley \& Smith (1972) explicam que, quando as variações de espaçamento para uma mesma espécie não são extremas, a altura final não é significativamente afetada pelo espaçamento, ao contrário do que ocorre com o crescimento em diâmetro.

Em termos de mortalidade, o comportamento esperado era de que os tratamentos submetidos às maiores intensidades de desbaste proporcionassem taxas mínimas de mortalidade (Kariuki, 2008) devido à redução da competição entre as plantas. Os resultados encontrados neste estudo diferiram, em parte, do esperado. A maior taxa de mortalidade foi obtida no $T 50$ com 7,08\% (Tabela 4) e a Tabela 3 mostra que no período imediatamente após o desbaste ocorreu a maior mortalidade de indivíduos neste tratamento (18 indivíduos).

Essa alta mortalidade de indivíduos pode ter sido causada devido ao impacto da colheita das árvores na implantação do desbaste neste tratamento. Como o desbaste foi realizado de forma sistemática e a remoção de árvores foi alternada dentro da mesma linha, aumentou-se a probabilidade de ocorrência de danos mecânicos aos indivíduos remanescentes, que pode ter levado ao aumento do número de árvores mortas neste tratamento.

Desconsiderando estes 18 indivíduos, a taxa de mortalidade ao final dos 35 meses para o tratamento $T 50$ seria em torno de 1,5\%, o menor entre os tratamentos e estaria coerente com o encontrado na literatura.

Nos demais períodos avaliados (Tabela 3), a mortalidade ocorreu de forma aleatória entre os tratamentos, sendo mais incidente no tratamento testemunha. Ramos et al. (2014) não encontraram diferenças na mortalidade entre povoamentos desbastados e não desbastados. Porém os autores constataram tendência de aumento da mortalidade para o povoamento não desbastado, indicando que a competição intraespecífica manteve-se elevada nas plantas daquele tratamento.

Com relação aos ajustes hipsométricos, percebe-se que os valores obtidos para o coeficiente de determinação $\left(R^{2}\right)$ variaram significativamente (Tabela 5). Essas variações observadas tanto nos valores de $R^{2}$ quanto para os valores de erro-padrão, indicam que a relação hipsométrica não é uma relação dendrométrica muito forte (Araújo et al., 2012), devido a alta variabilidade das alturas para uma mesma classe de diâmetro (Bartoszeck et al., 2004; Figueiredo Filho et al., 2010; Martins et al., 2016).

De maneira geral, os modelos hipsométricos proporcionaram ajustes satisfatórios e coerentes com os encontrados na literatura (Soares et al., 2004; Machado et al., 2008; David et al., 2016). As estatísticas de qualidade das estimativas, principalmente às da fase de validação foram muito similares, havendo superioridade do modelo 3 em relação ao demais.

A análise gráfica dos resíduos corrobora a superioridade indicada nas estatísticas de qualidade do modelo de Curtis devido à menor dispersão dos pontos, principalmente nas estimativas destinadas à validação dos modelos. Outros autores testando relações hipsométricas confirmaram o melhor desempenho deste modelo na estimação da altura total de árvores (Soares et al., 2004; Machado et al., 2008; Araújo et al., 2012; David et al., 2016).

O teste de Identidade de modelos apresentou-se significativo ao nível de 5\% na maioria das combinações entre os tratamentos testados (Tabela 6), indicando que não é possível aplicar uma única equação ajustada para todos os tratamentos testados. Para as comparações entre os tratamentos T0 e T25; TO e T50 aos 67 meses e, T0 e T25 aos 88 meses o teste foi não significativo e indica que é possível utilizar apenas uma equação para estimar a altura total das árvores destes tratamentos nas referidas idades. 
Conforme os resultados obtidos, os desbastes não afetaram significativamente o crescimento em altura, mas afetaram significativamente o crescimento em diâmetro (Hawley \& Smith, 1972). Essas alterações nos incrementos diamétricos resultam em variações na relação altura-diâmetro entre os tratamentos, o que explica os resultados obtidos no teste de identidade de modelos.

\section{CONCLUSÕES}

As intensidades de desbaste influenciam o crescimento em DAP e área basal por hectare, porém pouco influenciam o crescimento em altura total.

O modelo hipsométrico desenvolvido por Curtis proporciona estimativas consistentes da altura total das árvores, por idade e para os diferentes tratamentos.

As alterações nos incrementos diamétricos proporcionadas pelo desbaste impossibilitam a utilização de uma única equação hipsométrica para os diferentes tratamentos em cada idade.

\section{REFERÊNCIAS}

Alvares, C. A., Stape, J. L., Sentelhas, P. C., de Moraes Gonçalves, J. L., \& Sparovek, G. (2013). Köppen's climate classification map for Brazil. Meteorologische Zeitschrift (Berlin), 22(6), 711-728. http://dx.doi.org/10.1127/0941-2948/2013/0507.

Araújo, E. J. G., Pelissari, A. L., David, H. C., Scolforo, J. R. S., Péllico Netto, S., \& Morais, V. A. (2012). Relação hipsométrica para candeia (Eremanthus erythropappus) com diferentes espaçamentos de plantio em Minas Gerais, Brasil. Pesquisa Florestal Brasileira, 32(71), 257-268. http://dx.doi.org/10.4336/2012.pfb.32.71.257.

Araújo, L. V. C., Paulo, M. C. S., \& Paes, J. B. (2007). Características dendrométricas e densidade básica da Jurema-preta (Mimosa tenuiflora (Willd.) Poir.) de duas regiões do estado da Paraíba. Revista Caatinga, 20(1), 89-96.

Assmann, E. (1961). Waldertragskunde (435 p.). Muenchen: BLV Verlagsgessellschaft.

Azevedo, T. L., Mello, A. A., Ferreira, R. A., Sanquetta, C. R., \& Nakajima, N. Y. (2011). Equações hipsométricas e volumétricas para um povoamento de Eucalyptus sp. localizado na FLONA do Ibura, Sergipe. Agrária, 6(1), 105-112. http://dx.doi.org/10.5039/agraria.v6i1a861.

Bartoszeck, A. C., Machado, S. A., Figueiredo Filho, A., \& Oliveira, E. B. (2004). Dinâmica da relação hipsométrica em função da idade, do sítio e da densidade inicial de povoamentos de bracatinga da Região metropolitana de Curitiba, PR. Revista Árvore, 28(4), 517-533. http://dx.doi.org/10.1590/S0100-67622004000400006.

Binoti, M. L. M. S., Leite, H. G., Binoti, D. H. B., \& Gleriani, J. M. (2015). Prognose em nível de povoamento de clones de eucalipto empregando redes neurais artificiais. Cerne, 21(1), 97-105. http://dx.doi.org/10.1590/01047760201521011153.

Campos, J. C. C., \& Leite, H. G. (2017). Mensuração florestal: perguntas e respostas (5. ed., 636 p.). Viçosa: UFV, 2017.

Carneiro, J. G. A., Ferraz, T. M., Silva, M. P. S., \& Barroso, D. G. (2012). Princípios de desrama e desbastes florestais (96 p.). Viçosa: Editora UFV.

Curtis, R. (1967). Height-diameter and height-diameter-age equations for second-growth Douglas-fr. Forest Science, 13(4), 365-375. https://doi.org/10.1093/forestscience/13.4.365.

David, H. C., Arce, J. E., Péllico Netto, S., Corte, A. P. D., Marinheski Filho, A., \& Araújo, E. J. G. (2016). Efeito do desbaste e da qualidade do sítio na relação hipsométrica de Pinus taeda. Floresta e Ambiente, 23(2), 220-228. http://dx.doi.org/10.1590/2179-8087.081114.

David, H. C., Péllico Netto, S., Arce, J. E., Corte, A. P. D., Marinheski Filho, A., \& Araújo, E. J. G. (2017). Efeito da qualidade do sítio e do desbaste na produção de Pinus. Floresta e Ambiente, 24, e00096414. http://dx.doi.org/10.1590/2179-8087.096414.

Dias, A. N., Leite, H. G., Campos, J. C. C., Couto, L., \& Carvalho, A. F. (2005). Emprego de um modelo de crescimento e produção em povoamentos desbastados de Eucalipto. Revista Árvore, 29(5), 731-739. http://dx.doi.org/10.1590/S0100-67622005000500008. 
Donadoni, A. X., Pelissari, A. L., Drescher, R., \& Rosa, G. D. (2010). Relação hipsométrica para Pinus caribaea var. hondurensis e Pinus tecunumanii em povoamento homogêneo no Estado de Rondônia. Ciência Rural, 40(12), 2499-2504. http://dx.doi.org/10.1590/S0103-84782010001200010.

Figueiredo Filho, A., Dias, A. N., Kohler, S. V., Verussa, A. A., \& Chiquetto, A. L. (2010). Evolution of the hypsometric relationship in Araucaria angustifolia plantations in the mid-south region of Paraná State. Cerne, Lavras, MG, 16(3), 347-357. http://dx.doi.org/10.1590/S0104-77602010000300010.

Glencross, K., Palmer, G., Pelletier, M.-C., Doland Nichols, J., \& Smith, R. G. B. (2014). Basal area increment is unaffected by thinning intensity in young Eucalyptus dunnii and Corymbia variegata plantations across different quality sites. Forest Ecology and Management, 318, 326-333. http://dx.doi.org/10.1016/j.foreco.2014.01.041.

Glufke, C., Finger, C. A. G., \& Schneider, P. R. (1997). Crescimento de Pinus elliotti Engelm sob diferentes intensidades de desbaste. Ciência Florestal, 7(1), 11-26. http://dx.doi.org/10.5902/19805098335.

Gonçalves, F. G., Oliveira, J. T. S., Silva, G. F., Nappo, M. E., \& Tomazelo Filho, M. (2010). Parâmetros dendrométricos e correlações com propriedades tecnológicas em um híbrido clonal de Eucalyptus urophylla x Eucalyptus grandis. Revista Árvore, 34(5), 947-959. http://dx.doi.org/10.1590/S010067622010000500020.

Graybill, F. A. (1976). Theory and application of linear model (704 p.). Belmont: Duxbury.

Haridasan, M. (1990). Solos do Distrito Federal. In M. N. Pinto (Ed.), Cerrado: caracterização, ocupação e perspectivas (pp. 309-330). Brasília: Editora Universidade de Brasília.

Hawley, R. C., \& Smith, D. M. (1972). Silvicultura prática (544 p.). Barcelona: Omega.

Hawthorne, S. N. D., Lane, P. N. J., Bren, L. J., \& Sims, N. C. (2013). The long term effects of thinning treatments on vegetation structure and water yield. Forest Ecology and Management, 310, 983-993. http://dx.doi.org/10.1016/j.foreco.2013.09.046.

Hoffmann, R. G., Silva, G. F., Chichorro, J. F., Ferreira, R. L. C., Vescovi, L. B., \& Zaneti, L. Z. (2011). Caracterização dendrométrica de plantios de paricá (Schizolobium amazonicum Huber ex. Ducke) na região de Paragominas, PA. Agrária, 6(4), 675-684. http://dx.doi.org/10.5039/agraria.v6i4a1039.

Hsing, T. Y., Paula, N. F., \& Paula, R. C. (2016). Características dendrométricas, químicas e densidade básica da madeira de híbridos de Eucalyptus grandis X Eucalyptus urophylla. Ciência Florestal, 26(1), 273-283. http://dx.doi.org/10.5902/1980509821119.

Husch, B., Beers, T. W., \& Kershaw Junior, J. A. (2003). Forest mensuration (4th ed., 443 p.). Hoboken, New Jersey: John Wiley \& Sons.

Kariuki, M. (2008). Modelling the impacts of various thinning intensities on tree growth and survival in a mixed species eucalypt forest in central Gippsland, Victoria, Australia. Forest Ecology and Management, 256(12), 2007-2017. http://dx.doi.org/10.1016/j.foreco.2008.07.035.

Leite, H. G., Nogueira, G. S., Campos, J. C. C., Souza, A., \& Carvalho, A. (2005). Avaliação de um modelo de distribuição diamétrica ajustado para povoamentos de Eucalyptus sp. submetidos a desbaste. Revista Árvore, 29(2), 271-280. http://dx.doi.org/10.1590/S0100-67622005000200010.

Lima, I. L., \& Garcia, J. N. (2011). Efeito do desbaste e da fertilização na porcentagem de casca e conicidade de toras de Eucalyptus grandis. Floresta, 41(2), 305-312. http://dx.doi.org/10.5380/rf.v41i2.21878.

Machado, S. A., Nascimento, R. G. M., Augustinczik, A. L. D., Silva, L. C. R., Figura, M. A., Pereira, E. M., \& Teo, S. J. (2008). Comportamento da relação hipsométrica de Araucaria angustifolia no capão da Engenharia Florestal da UFPR. Pesquisa Florestal Brasileira, (56), 5-16.

Martins, E. R., Binoti, M. L. M. S., Leite, H. G., Binoti, D. H. B., \& Dutra, G. C. (2016). Configuração de redes neurais artificiais para estimação da altura total de árvores de eucalipto. Agrarian (Dourados), 11(2), 117-123. http://dx.doi.org/10.5039/agraria.v11i2a5373.

Monte, M. A., Reis, M. G. F., Reis, G. G., Leite, H. G., Cacau, F. V., \& Alves, F. F. (2009). Crescimento de um clone de eucalipto submetido a desrama e desbaste. Revista Árvore, 33(5), 777-787. http://dx.doi.org/10.1590/S0100-67622009000500001.

Neter, J., Kutner, M. H., Nachtsheim, C. J., \& Wasserman, W. (1996). Applied linear statistical models (4th ed., 1408 p.). Chicago: Irwin.

Nimer, E. (1989). Climatologia do Brasil (422 p.). Rio de Janeiro: Departamento de Recursos Naturais e Estudos Ambientais, Instituto Brasileiro de Geografia e Estatistica.

Ozçelik, R., Diamantopoulou, M. J., Brooks, J. R., \& Wiant Junior, H. V. J. (2010). Estimating tree bole volume using artificial neural network models for four species in Turkey. Journal of Environmental Management, 91(3), 742-753. PMid:19880241. http://dx.doi.org/10.1016/j.jenvman.2009.10.002. 
Ramos, D. C., Reis, G. G., Reis, M. G. F., Leite, H. G., Stocks, J. J., Lima, A. P. L., \& Faria, R. S. (2014). Thinning effect on plant growth of pruned eucalyptus clone. Revista Árvore, 38(3), 495-503. http://dx.doi.org/10.1590/S0100-67622014000300012.

Regazzi, A. J. (2003). Teste para verificar a igualdade de parâmetros e a identidade de modelos de regressão não-linear. Revista Ceres, 50, 9-26.

Soares, A. A. V., Leite, H. G., Cruz, J. P., \& Forrester, D. I. (2017). Development of stand structural heterogeneity and growth dominance in thinned Eucalyptus stands in Brazil. Forest Ecology and Management, 384, 339-346. http://dx.doi.org/10.1016/j.foreco.2016.11.010.

Soares, C. P. B., Neto, F. P., \& Souza, A. L. (2011). Dendrometria e inventário florestal (2. ed., 272 p.). Viçosa: UFV.

Soares, T. S., Scolforo, J. R. S., Ferreira, S. O., \& Mello, J. M. (2004). Uso de diferentes alternativas para viabilizar a relação hipsométrica no povoamento florestal. Revista Árvore, 28(6), 845-854. http://dx.doi.org/10.1590/S0100-67622004000600009.

Stoffels, A., \& Soest, J. van (1953). The main problems in sample plots. Ned Bosbouwtijdschr, 25, 190-199.

Sullivan, T. P., Sullivan, D. S., Lindgren, P. M. F., \& Ransome, D. B. (2006). Long-term responses of ecosystem components to stand thinning in young lodgepole pine forest III. Growth of crop trees and coniferous stand structure. Forest Ecology and Management, 228(1-3), 69-81. http://dx.doi.org/10.1016/j.foreco.2006.02.026.

Trevisan, R., Haselein, C. R., Santini, E. J., Schneider, P. R., \& Menezes, L. F. (2007). Efeito da intensidade de desbaste nas características dendrométricas e tecnológicas da madeira de Eucalyptus grandis. Ciência Florestal, 17(4), 377-387. http://dx.doi.org/10.5902/198050981969.

Vidaurre, G. B., Nutto, L., França, F. J. N., Braz, R. L., Watzlawick, L. F., \& Moulin, J. C. (2015). Tensão de crescimento no lenho de Eucalyptus benthamii e sua relação com características dendrométricas em diferentes espaçamentos. Floresta e Ambiente, 22(3), 408-415. http://dx.doi.org/10.1590/21798087.044413.

Contribuição dos Autores: LSMJ: conceituação, curadoria de dados, análise formal, investigação, metodologia, administração do projeto, software, validação, escrita - primeira redação, escrita - revisão e edição; RVOC: conceituação, análise formal, obtenção de financiamento, investigação, metodologia, administração do projeto, recursos, supervisão, validação, visualização, escrita - revisão e edição; MEN: conceituação, análise formal, metodologia, investigação, administração do projeto, recursos, supervisão, validação, visualização, escrita revisão e edição; GSN, CAAJ e ROG: metodologia, visualização, escrita - revisão e edição; LSC e EKBO: curadoria de dados, visualização, escrita - revisão e edição. 